\title{
Enhanced Environmental Design for a New Integrated Hyper-Modal Microscope
}

\author{
David C. Bell ${ }^{1}$, Adam Graham ${ }^{2}$, Joe Rooney ${ }^{3}$, Larry Cessna ${ }^{3}$, Joseph D. Gibbons ${ }^{4}$, Heiko Müller ${ }^{5}$ and \\ Maximilian Haider ${ }^{5}$ \\ 1. Harvard John A. Paulson School of Engineering and Applied Sciences, Harvard University, \\ Cambridge, MA, USA \\ 2. Center for Nanoscale Systems, Harvard University Cambridge MA, USA \\ 3. Hitachi High Technologies America, Inc. \\ 4. Wilson Architects Inc, Boston MA \\ 5. CEOS Gmbh, Heidelberg Germany
}

Though the resolution of electron microscopy has increased to a sub-Angstrom level, even a small environmental perturbation will cause loss of resolution. Coupled to low accelerating voltage operation for high resolution EELS with possible monochromated electron energy sources, we can measure quantities such as optical resonances (Fig. 1.) and inter and intra band transitions below the Cherenkov radiation background [1]. These types of measurements require a total systematic design approach that will allow all parts of the instrument to operate within a cocoon of high stability. The new microscope design will include multiple functionality incorporating very long beam path that would be impacted by environmental noise. The physical conditions of the lab room where the electron microscope resides could impact the device by vibration, fluctuating temperature, high humidity and electromagnetic fields. The impacting factors can be classified into three main categories: 1) factors that change the relative position between samples and the probe, 2) factors that affect the detection sensitivity of the probe, and 3) factors that might contaminate or modify the samples.

Vibration and thermal fluctuation are the main factors that change the relative position between samples and the probe. A vertical vibration will cause the focus zone to spread and reduce its sharpness of resolution. A horizontal vibration causes the captured image blurring since multiple areas might overlap which reduces the resolution. The sources of vibration might come from the vibration of ancillary equipment inside the room, and outside the room, e.x. people walking in the upper floor, earthquake, passing by vehicles and acoustic vibration. Thermal fluctuation shifts the image by changing sample shape or size, e.x. expansion and shrinkage. Thermal fluctuation could be the result of improper controlled air conditioning system. Electrons could deflect under magnetic and electric field, thus a noisy electromagnetic background will distort the probing current, lead to aberration and reduce the resolution. The source of electromagnetic interferences might come from the power cable, a display and devices neighboring spaces. Effective passive and dynamic shielding approaches are taken to minimize such impacts.

Both the microscope and its environment are designed to maximize the advances in new electron optics [2] such as fifth order aberration corrected optics for high resolution imaging and dynamic analytical methods designed and implemented by CEOS. Hyper-modal operation of the microscope is now realized by integrated high speed camera systems for the imaging of dynamic processes as well as new analytical detectors such as large area EDS systems and new high energy resolution EELS systems. The instrument can also be used for environmental imaging in a gas flow environment by use of a gas injection system. Coupled with the ability for high resolution holography and Lorentz modes the total system (both the instrument and the room) have been designed to allow operation of all functionalities at the highest level of performance. With the introduction of simultaneous atomic-resolution electron ptychography and Z-contrast imaging of light and heavy elements in complex nanostructures allows for truly atomic-scale functional imaging in aberration-corrected electron microscopy [3]. 
References:

[1] D.C. Bell, C.J. Russo and D. Kolmykov, Ultramicroscopy 114 (2012), p. 31.

[2] S. Uhlemann et al, Phys. Rev. Lett. 111, p. 046101

[3] This work was supported by the STC, Center for Integrated Quantum Materials, NSF Grant No. DMR1231319. This work was performed at the Center for Nanoscale Systems (CNS), a member of the National Nanotechnology Coordinated Infrastructure Network (NNCI), which is supported by the National Science Foundation under NSF award no. 1541959.
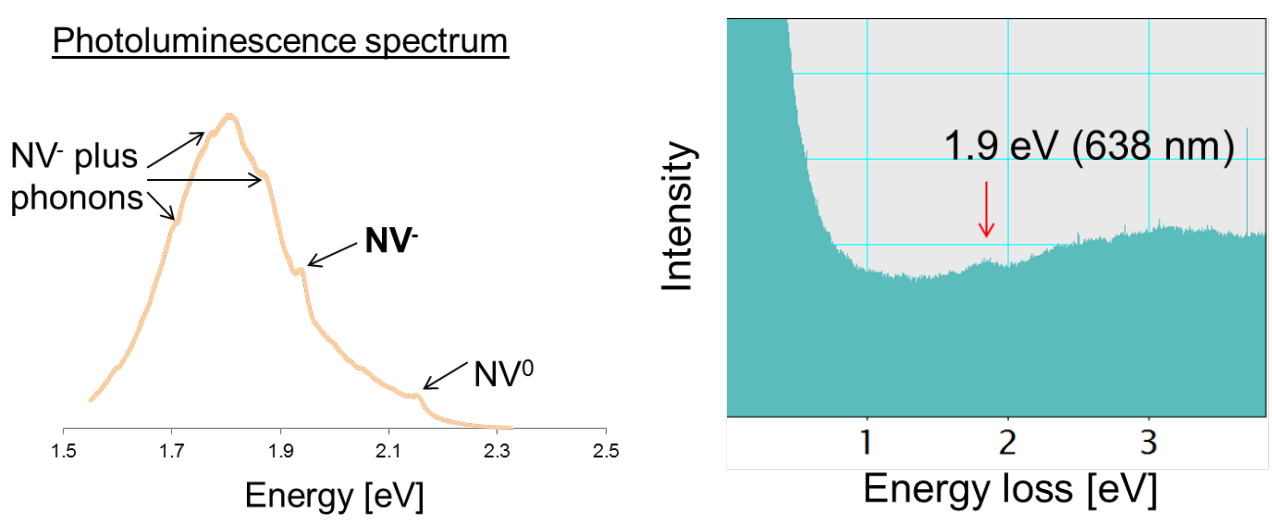

Figure 1. Extreme LV-EELS of Nitrogen Vacancy centers in diamond. Left, the corresponding PL spectra of NV centers in a sample of thin diamond. Right, the Low Voltage Monochromated LV-EELS spectra from the same sample showing NV signal.

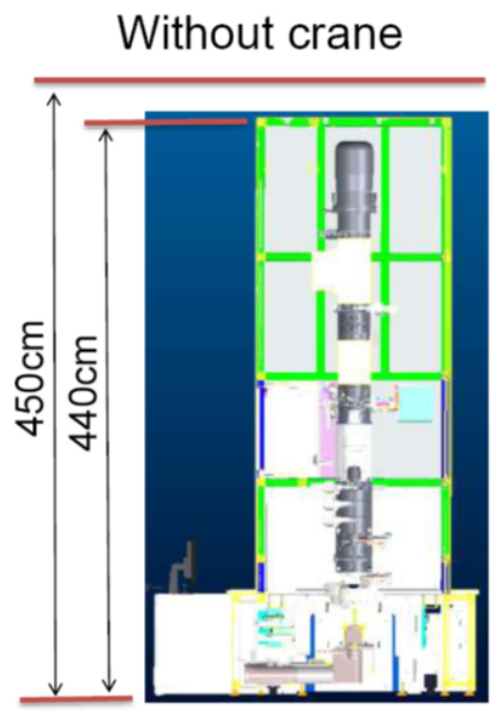

Figure 2. Column length of the Hitachi HF3300S, illustrates one of the challenges for engineering design for instrument stability and room design specification. 